\title{
Temperature effects on multi-particle scattering in a gapped quantum magnet
}

\author{
S. Notbohm ${ }^{\mathrm{a}, \mathrm{b}}$, D.A. Tennant ${ }^{\mathrm{a}, \mathrm{c}}$, B. Lake ${ }^{\mathrm{a}, \mathrm{c}, \mathrm{d}}$, P.C. Canfield ${ }^{\mathrm{d}}$, J. Fielden ${ }^{\mathrm{d}}$, P. Kögerler ${ }^{\mathrm{d}}$ \\ , H.-J. Mikeska ${ }^{\mathrm{e}}$, C. Luckmann ${ }^{\mathrm{e}}$, M.T.F. Telling ${ }^{\mathrm{f}}$ \\ ${ }^{a}$ Hahn-Meitner-Institut, Glienicker Straße 100, 14109 Berlin, Germany \\ ${ }^{\mathrm{b}}$ School of Physics and Astronomy, University of St. Andrews, North Haugh, St. Andrews, Fife, KY16 9SS, U.K. \\ ${ }^{\mathrm{c}}$ Institut für Festkörperphysik, Technische Universität Berlin, Hardenbergstr. 36, 10623 Berlin, Germany \\ ${ }^{\mathrm{d}}$ Ames Laboratory and Department of Physics and Astronomy, Iowa State University, Ames, Iowa, 50011, USA \\ ${ }^{\mathrm{e}}$ Institut für Theoretische Physik, Universität Hannover, Appelstr. 2, 30167 Hannover, Germany \\ ${ }^{\mathrm{f}}$ ISIS Facility, Rutherford Appleton Laboratory, Chilton, Didcot OX11 OQX, U.K.
}

Received 13 December 2006

\begin{abstract}
We report measurements of the temperature effects on the dimerized antiferromagnetic chain material, copper nitrate $\mathrm{Cu}\left(\mathrm{NO}_{3}\right)_{2} \cdot 2.5 \mathrm{D}_{2} \mathrm{O}$. Using inelastic neutron scattering we have measured the temperature dependence of the one- and two-magnon excitation spectra as well as the temperature induced one-magnon intra-band scattering in a single crystal. Comparison is made with numerical evaluations of thermal averages based on the calculation for a chain of 16 spins.
\end{abstract}

(c) 2006 Elsevier B.V. All rights reserved.

PACS: $78.70 . \mathrm{Nx}$ 75.30.Ds $75.10 . \mathrm{Jm}$

Keywords: Quantum Magnet; Dynamics; Neutron Scattering

Low-dimensional quantum antiferromagnets serve as model systems to investigate the effects of quantum fluctuations. In contrast to one-magnon states, higher order excitations and temperature effects have received less attention. These, however, will give interesting insight to the interplay between thermal and the quantum fluctuations in gapped quantum magnets $[1,2]$. One particularly tractable case is the alternating Heisenberg antiferromagnetic chain (AHC) with spin 1/2 [3]. Copper nitrate is especially attractive because the alternation parameter of $\alpha \approx 0.24$ $\left(\alpha \equiv J_{2} / J_{1}\right)$ ensures an energy separation between the different scattering processes [4] (see Fig. 1). Motivated by this we have measured the temperature dependence of the one-, two-magnon, and additionally intra-band scattering processes using newly available low background and high resolution time-of-flight spectroscopy.

The $\Delta S=1$ excitation spectrum of the chain is displayed in Fig. 1. The AHC has a gap to its one-magnon dispersion (solid curve) and another gap to the two-magnon continuum (dashed curves) accessible by neutron scattering via vacuum fluctuations. At certain wave-vectors where the interaction potential is strong two magnons can lower their energy by forming a pair, known as bound mode [1]. Additionally a new continuum centered around zero energy arises with temperature (dashed curves) from intra-band

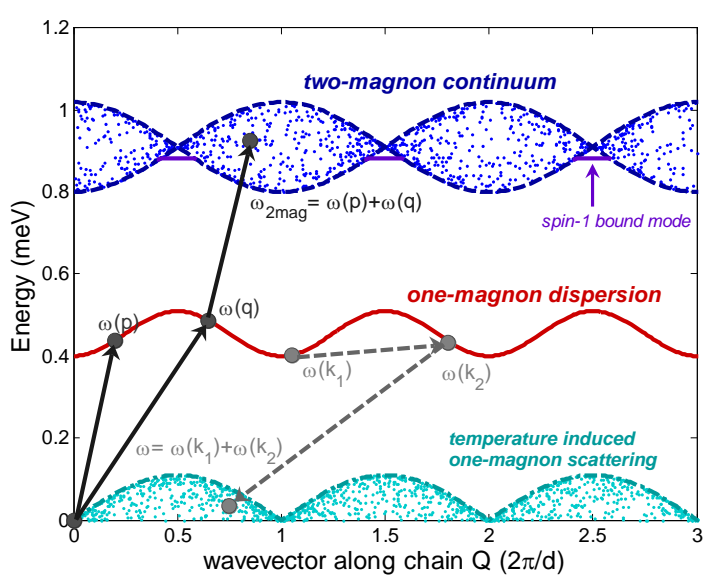

Fig. 1. $\Delta S=1$ excitation spectrum for a dimerized chain. The gapped one-magnon band and the two magnon continuum are shown and the scattering of two magnons illustrated by black arrows. When applying temperature to the system one-magnon intra-band scattering centered at zero energy occurs shown by arrows and bounded by grey the dashed curve.

scattering. It can be constructed by moving a magnon along the dispersion curve to a new wave-vector. The effect of this quasi-elastic scattering is similar to the Villain mode scattering of soliton systems [5]. 


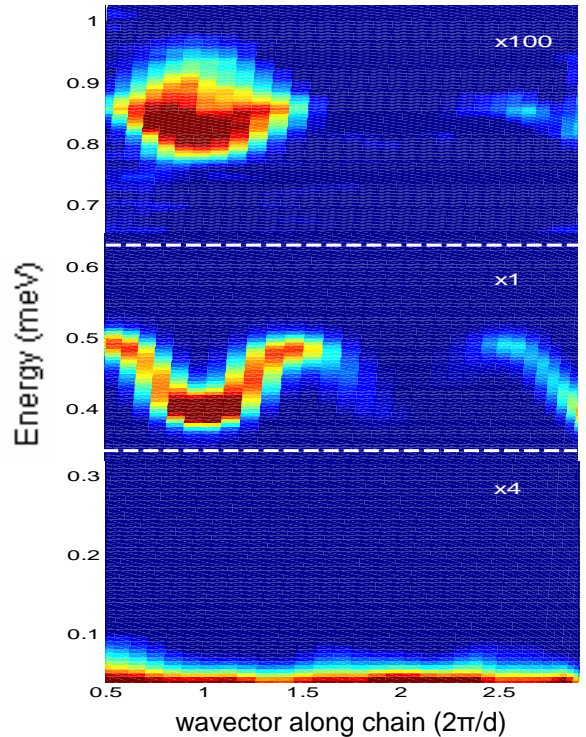

Fig. 2. The measured excitation spectrum with non-magnetic background subtracted and corrections for instrumental resolution and form factor. One- and two-magnon data obtained at $120 \mathrm{mK}$, intra-band scattering at $6 \mathrm{~K}$. Two-magnon scattering enhanced by a factor of 100, intra-band scattering by 4 .

A $8 g$ single crystal of $\mathrm{Cu}\left(\mathrm{NO}_{3}\right)_{2} \cdot 2.5 \mathrm{D}_{2} \mathrm{O}$ was grown out of a $\mathrm{D}_{2} \mathrm{O}$ solution that had been enriched in $\mathrm{D}$ over $\mathrm{H}$ to a deuteration level of $98 \%$ by a successive evaporation and dilution. The inelastic neutron scattering measurements were performed using the OSIRIS time-of-flight back-scattering spectrometer at ISIS, Rutherford Appleton Laboratory, U.K. The sample was mounted in a dilution insert giving base temperature of $120 \mathrm{mK}$ and oriented with the chain wave-vector $Q$ perpendicular to the incident neutron wave-vector $k_{i}$. The magnetic cross-section was normalized using incoherent nuclear scattering from a vanadium standard. Data were collected for a final energy $E_{f}=$ $1.845 \mathrm{meV}$ at $0.12,2,4$ and $6 \mathrm{~K}$.

The non-magnetic background for the one-magnon signal was corrected using an energy cut of $Q=2.1 \pm 0.05$ where the magnetic scattering vanished due to the dimer form factor $1-\cos (q \cdot d)$ and any remaining signal results from incoherent scattering. For the two-magnon a Gaussian centered at $0.876 \mathrm{meV}$ with width and amplitude determined from the $Q=2.1 \pm 0.05$ cut was used to account for the incoherent signal. For the intra-band scattering the $120 \mathrm{mK}$ data served as incoherent background. The background subtracted data is displayed in Fig. 2.

Fig. 3 shows the evolution of the temperature dependence for the excitation spectrum. The data is compared to a numerical evaluation of thermal averages. The exact temperature dependence is calculated for all eigenvalues for chains of 16 spins [2]. These calculations are displayed for 2, 4 and $6 K$ and show good agreement with our measurements. Fig.3 (a) shows the intra-band scattering taken for a wavevector $Q=0.5 \pm 0.05$ at intra-band maximum (see Fig. 1). Fig. 3 (b) and (c) show the one-and the two-magnon tem-

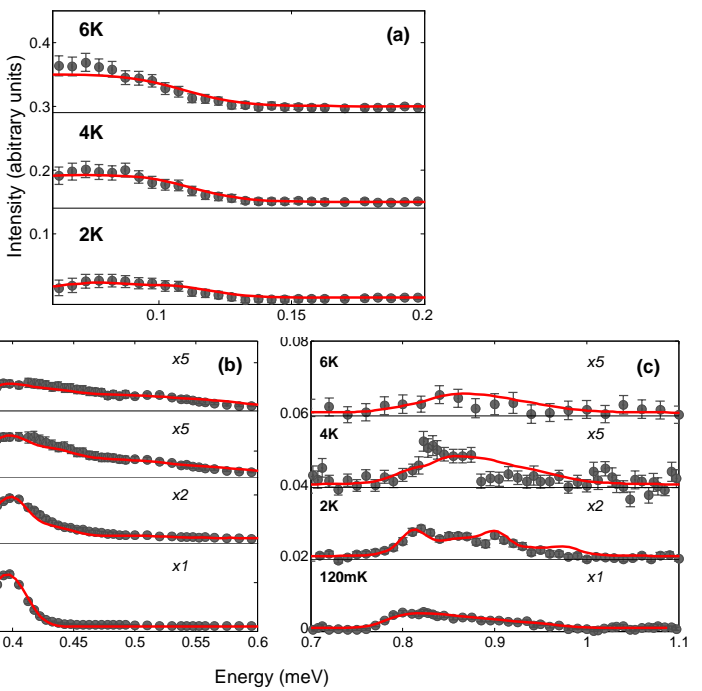

Fig. 3. Comparison of theory and background-subtracted data. (a) Intra-band scattering displayed for 2,4 and $6 \mathrm{~K}$. (b) One-magnon scattering for $120 \mathrm{mK}, 2,4$ and $6 \mathrm{~K}$. Intensities of data and model at $2 \mathrm{~K}$ enhanced by a factor of 2 , and at 4 and $6 \mathrm{~K}$ by a factor of 5 to display everything on the same scale. (c) Two-magnon sacttering. Lines represent theoretical fit to data. A wave-vector dependent shift is added to the theroretical function to account for the interchain coupling.

perature development respectively. Here the cut was taken around $Q=1$. The $120 \mathrm{mK}$ curve for the one-magnon data is the dispersion described by $\mathrm{Xu}$ [3], while the two-magnon is based on a $1 \mathrm{D}$ perturbation theory approach [1] with coupling parameters $J=0.446 \mathrm{meV}$ and alternatation parameter $\alpha=0.24$. The calculated intensities of the intraband scattering had to be enhanced by a factor of 1.7 to match the data. This suggests that calculations involving 16 spins might not be sufficient and analytical calculations necessary.

In conclusion we have a clean measurement of the excitation spectrum of an AHC over a range of temperatures. The one-magnon, two-magnon and for the first time the intra-band scattering have been analyzed and compared to theory. In general there is a good agreement between data and theory which can be compared by using more accurate values of $J$ and $\alpha$, including exact dimer distances and the weak intra-chain coupling.

Ames Laboratory is operated for the U.S. Department of Energy by Iowa State University under Contract No. W7405-ENG-82. This work was supported by the Director for Energy Research, Office of Basic Energy Sciences. S.N. would like to thank M. Angst for his help during the growth process.

\section{References}

[1] D.A. Tennant et al., Phys. Rev. B 67, 054414 (2003).

[2] H.-J. Mikeska and C. Luckmann, Phys. Rev. B 73, 184426 (2006).

[3] G. Xu et al., Phys. Rev. Lett. 84, 4465 (2000). 
[4] T. Barnes, J. Riera, and D.A. Tennant, Phys. Rev. B 59, 11384 (1999).

[5] J. Villain, Physics D 79,1 (1975). 